\title{
Design and Analysis of Separate-Absorption-Transport-Charge-Multiplication Traveling-Wave Avalanche Photodetectors
}

\author{
Jin-Wei Shi, Yin-Hsin Liu, and Chee-Wee Liu, Senior Member, IEEE
}

\begin{abstract}
This paper proposes a novel type of avalanche photodiode - the separate-absorption-transport-charge-multiplication (SATCM) avalanche photodiode (APD). The novel design of photoabsorption and multiplication layers of APDs can avoid the photoabsorption layer breakdown and hole-transport problems, exhibit low operation voltage, and achieve ultra-high-gain bandwidth product performances. To achieve low excess noise and ultra-high-speed performance in the fiber communication regime $(1.3 \sim 1.55 \mu \mathrm{m})$, the simulated APD is Si-based with an SiGe-Si superlattice (SL) as the photoabsorption layer and traveling-wave geometric structures. The frequency response is simulated by means of a photo-distributed current model, which includes all the bandwidth-limiting factors, such as the dispersion of microwave propagation loss, velocity mismatch, boundary reflection, and multiplication/transport of photogenerated carriers. By properly choosing the thicknesses of the transport and multiplication layers, microwave propagation effects in the traveling-wave structure can be minimized without increasing the operation voltage significantly. A near 30-Gb/s electrical bandwidth and $10 \times$ avalanche gain can be achieved simultaneously, even with a long device absorption length $(150 \mu \mathrm{m})$ and low operation voltage $(\sim 12 \mathrm{~V})$. In addition, the ultrahigh output saturation power bandwidth product of this simulated TWAPD structure can also be expected due to the large photoabsorption volume and superior microwave-guiding structure.
\end{abstract}

Index Terms-Avalanche photodetector, avalanche photodiode (APD), high gain-bandwidth-product avalanche photodetector, high-saturation-power photodetector, $\mathrm{Si}$-SiGe-based photodetector, Si-SiGe-based superlattice (SL), traveling-wave photodetector, ultra-high-speed photodetector.

\section{INTRODUCTION}

$\mathbf{T}$ HE rapid growth in Internet traffic has created an urgent need to increase the channel bandwidth capacity of fiber-optic communication systems. As a key component in these transmission systems, ultra-high-speed photodetectors are able to not only increase the transmission capability in a single channel, but also reduce the required number of channels and complexity of management in a wavelength-division-multiplexing (WDM) fiber communication system [1]. There

Manuscript received May 29, 2003; revised February 4, 2004. This work was supported in part by the National Science Council of Taiwan under Grant NSC 92-2218-E-008-011.

J.-W. Shi is with the Department of Electrical Engineering, National Central University, Taoyuan 320, Taiwan (e-mail: jwshi@ee.ncu.edu.tw).

Y.-H. Liu and C.-W. Liu are with the Department of Electrical Engineering and the Graduate Institute of Electronic Engineering, National Taiwan University, Taipei 10617, Taiwan, and also with the Electronics Research and Service Organization (ERSO), Industrial Technology Research Institute (ITRI), Hsinchu 31040, Taiwan.

Digital Object Identifier 10.1109/JLT.2004.829230 are two major trends in the development of these high-speed photodetectors: 1) to obtain high-bandwidth-efficiency (sensitivity) products and 2) to improve the saturation output current under intense light illumination [2]. To achieve high-bandwidth-efficiency product performance, avalanche photodiodes (APDs) are preferred due to their internal gain and high sensitivity as compared with p-i-n photodiodes [3]-[6]. Ultra-high gain-bandwidth products with low excess noise performance of APDs have been demonstrated [4], [7] by optimizing the material [7], [8] or epilayer structures [4], [9], [10] of the multiplication layers. To obtain a high output saturation current, several photodetector technologies based on properly designed edge-coupled structures have been demonstrated to enhance power-bandwidth products. By reducing the optical modal absorption constant [11] or by achieving uniform photoabsorption [12] in edge-coupled structures with long device-absorption lengths, the output saturation current can be improved in a waveguide photodetector (WGPD) or traveling-wave photodetector (TWPD) [11], [13]. To avoid serious electrical bandwidth degradation in these devices having large areas (length), superior microwave-guiding electrode structures are necessary. Recently, record-high-peak output-power-bandwidth-product performance has been achieved by metal-semiconductor-metal TWPD structures [14].

In this paper, we propose a novel type of avalanche photodetector with a traveling-wave geometric structure, i.e., separate-absorption-transport-charge-multiplication (SATCM) traveling-wave avalanche photodiode (TWAPD). By incorporating novel epilayer structures of APDs and the geometry structure of the TWPD, the proposed devices can achieve superior microwave-guiding property, large photoabsorption volume with high power-bandwidth product, and high-gain-bandwidth-product performances. The photo-distributed current model is used to simulate the frequency response of the device [15]. This model can easily take into account all the microwave propagation effects and the internal response time of photogenerated carriers [15], and its accuracy has also been verified by means of experiments [16]. The modeled epilayer structures of APDs are Si-based with an SiGe-Si superlattice (SL) photoabsorption layer for telecommunication wavelength (1.3 $\sim 1.55 \mu \mathrm{m})$ applications [17], [18]. Conventional Si-based APDs exhibit much lower multiplication noise, shorter multiplication time, and larger gain-bandwidth product [7], [8], [19] than III-V-based APDs, because Si has very dissimilar electron $(\alpha)$ and hole $(\beta)$ impact ionization coefficients, and the ratio $(\beta / \alpha)$ of these coefficients is the lowest among any reported 


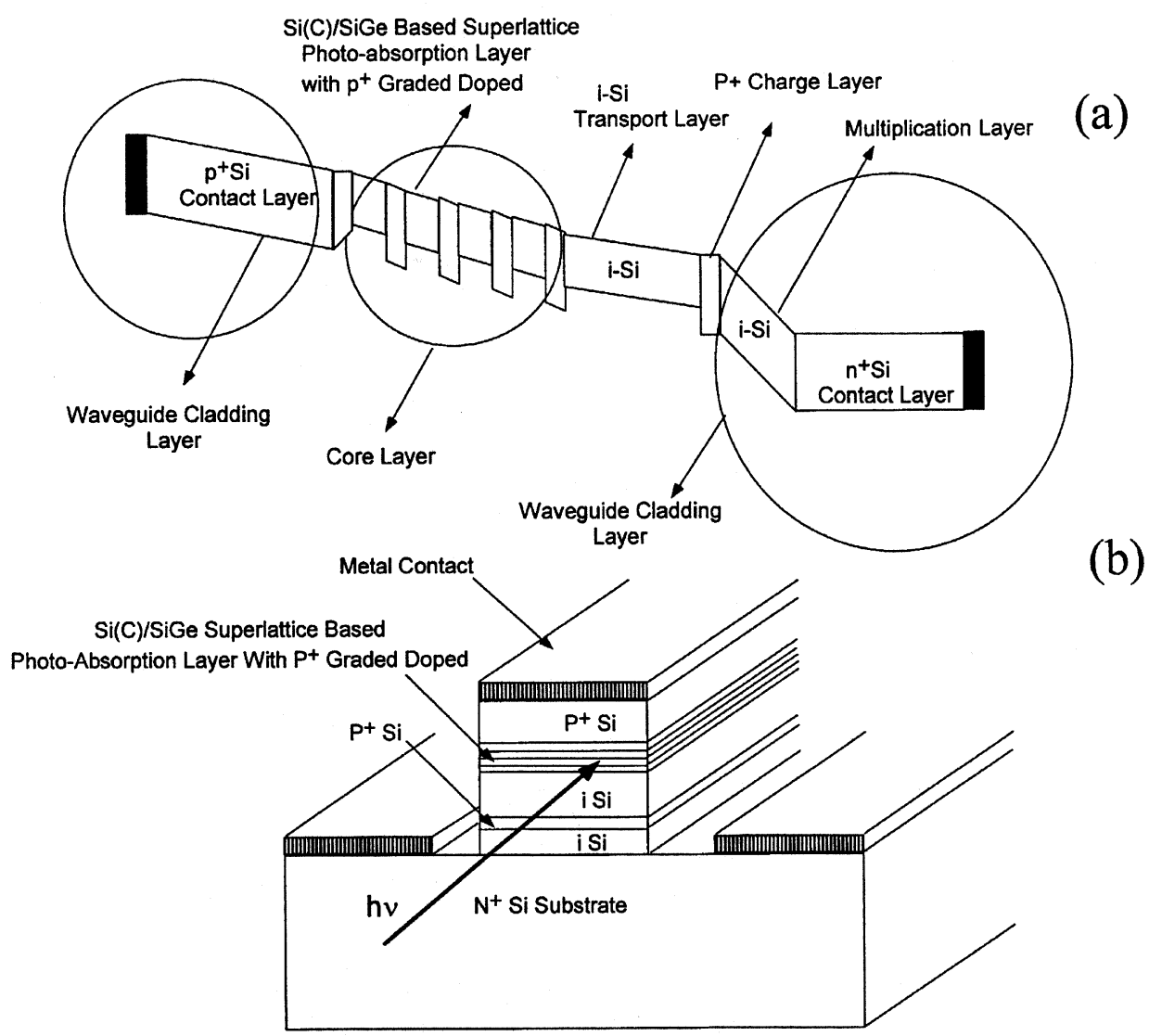

Fig. 1. (a) Conceptual band diagram and (b) cross-sectional view of the Si(C)-SiGe-based SATCM TWAPD.

semiconductor materials [19]. However, the wide absorption bandgap $(\sim 1.1 \mu \mathrm{m})$ of $\mathrm{Si}$ prevents it from being applied to long-wavelength $(1.3 \sim 1.55 \mu \mathrm{m})$ optical communications. Recently, several research groups have demonstrated the excellent noise and gain-bandwidth-product (GBP) performance of InGaAs-Si-heterostructure-based APDs by using a wafer-bonding technique in the long-optical-communication-wavelength regime [7], [8], [19]. The incorporation of the SiGe alloy on the $\mathrm{Si}$ substrate is another way to achieve long-wavelength absorption in Si-based photodetectors [17], [18], [20]-[26]. However, the demonstrated quantum efficiency of these photodetectors is much lower than the reported values of III-V-based photodetector's due to the relatively small photoabsorption constant of the strained SiGe alloy and the critical thickness constraint. The drawback of a small photoabsorption constant can be compensated by properly increasing the device-absorption length in the edge-coupled structure [18], [21]-[23], [27]. In addition, the low optical modal absorption constant also implies a more uniform and diluted photoabsorption process, lower density of photogenerated carriers, and higher output saturation power [11], [13], [27], [28]. According to our simulation results, this novel structure can exhibit $\sim 30-\mathrm{Gb} / \mathrm{s}$ electrical bandwidth and $\sim 10 \times$ avalanche gain simultaneously under low operation voltage $(\sim 12 \mathrm{~V})$ even with a long absorption length $(150 \mu \mathrm{m})$. The proposed device structure and the implication of the simulation results in this paper can not only be applied to the example $\mathrm{Si}-\mathrm{SiGe}$-based APD, but also serve as general guidelines for designing III-V-based TWAPDs or waveguide APDs
(WGAPDs) [29]-[31] with long device length, high-speed and high-saturation output power, and low operation voltage.

\section{Device Structure}

The conceptual band diagram and cross-sectional view of the proposed device are shown in Fig. 1(a) and (b), respectively. All of the important parameters used in our simulation are given in Table I. As shown in Fig. 1(b), three metal electrodes collect the photocurrent and act together as a coplanar waveguide (CPW) to support a photo-excited microwave-guiding mode. The optical wave-guiding structure is implemented using a ridge optical waveguide as in regular p-i-n TWPD [32]. The topmost $\mathrm{p}^{+}$-doped $\mathrm{Si}$ layer and $\mathrm{n}^{+}$-doped $\mathrm{Si}$ substrate serve as the cladding layers. The core layer, which is sandwiched between two cladding layers and acts as a photoabsorption layer, is composed of Si-SiGe SL. Si-SiGe quantum-well (QW)-based photodetectors with thick (over $\sim$ hundreds of $\AA$ ) barriers usually suffer from the hole-trapping problem under high-speed operation [20], [25]. In our design, we assume that the thickness of the barrier and well of the SiGe-Si SL are thin enough $(\leq 33 \AA$ Å) [33] to minimize this problem. The carbon-doped or undulating growth technique can also be applied in the SL to further increase its critical thickness [23], [25], [26], [34]. The composition of the simulated $\mathrm{SL}$ is $\mathrm{Si}_{0.5} \mathrm{Ge}_{0.5}-\mathrm{Si}$ to achieve an appropriate value of the optical absorption constant $\left(100 \mathrm{~cm}^{-1}\right)$ in the long-wavelength regime $(\sim 1.3 \mu \mathrm{m})$ [17], [26]. In traditional separate-absorption-charge-multiplication 
TABLE I

SIMULATION PARAMETERS

\begin{tabular}{|c|c|}
\hline & SATCM-TWAPD \\
\hline Center waveguide width & $3 \mu \mathrm{m}$ \\
\hline Ground plane width & $50 \mu \mathrm{m}$ \\
\hline $\mathrm{Si}(\mathrm{C}) / \mathrm{Si}_{0.5} \mathrm{Ge}_{0.5} \mathrm{SL}$ thickness & $200 \mathrm{~nm}$ \\
\hline $\mathrm{CPW}$ air gap & $2 \mu \mathrm{m}$ \\
\hline $\mathrm{P}^{+}$doped Si top layer thickness & $700 \mathrm{~nm}$ \\
\hline $\mathrm{P}^{+}$doped Charge layer thickness & $25 \mathrm{~nm}$ \\
\hline Transport layer thickness & $0 \mathrm{~nm} \sim 500 \mathrm{~nm}$ \\
\hline Metal (Al) thickness & $1 \mu \mathrm{m}$ \\
\hline Multiplication layer thickness & $100 \mathrm{~nm} \sim 500 \mathrm{~nm}$ \\
\hline $\begin{array}{c}\text { Resistivity of top and bottom } \mathrm{Si} \\
\text { layesr }\end{array}$ & $100 \Omega-\mu \mathrm{m}$ \\
\hline $\begin{array}{c}\text { Optical absorption constant }\left(\alpha_{v}\right) \text { of } \\
\mathrm{Si} / \mathrm{SiGe} \mathrm{SL}\end{array}$ & $100 \mathrm{~cm}^{-1}$ \\
\hline Device-absorption-length & $300 \mu \mathrm{m}$ and $150 \mu \mathrm{m}$ \\
\hline
\end{tabular}

(SACM) APDs, the photoabsorption layers are lightly and uniformly doped. Under avalanche operation, these layers suffer parts of applied bias voltages and are fully depleted [3], thereby enhancing the drift velocities of both photogenerated electrons and holes. However, under such high-bias voltage, these layers might suffer from the avalanche breakdown problem [3], [35], which can seriously degrade the GBP performance of APDs [3], [35]. As shown in Fig. 1(a), the photoabsorption layer in our proposed structure is the $\mathrm{p}^{+}$heavily graded doped SL, which can not only prevent the absorption layer breakdown, but also eliminate transport problems in photogenerated holes [2]. The multiplication layer is made of $\mathrm{Si}$, which is sandwiched in between an $\mathrm{n}^{+}$Si substrate and a $\mathrm{p}^{+}$charge layer [3], which serves as an electric-field buffer layer [32] to provide better control of the electric-field profile. Only photogenerated electrons form the $\mathrm{p}^{+}$-graded doped photoabsorption layer will drift through the transport layer and initiate the impact ionization process in the multiplication layer. The appropriate magnitude of the electric field must be located in these two layers to obtain a short drift time of photogenerated electrons and to initiate the avalanche breakdown process, respectively. In order to further reduce the operation voltage and multiplication time of APDs, the multiplication layer must be thin enough [3], [4], [19], [29], [37]. However, this approach will increase the device capacitance and reduce the microwave propagation velocity, which will limit the speed performance seriously, especially for the long-absorption-length TWPDs [15]. With an additional transport layer in our novel structure as shown in Fig. 1(a), the tradeoff between operation voltage and device capacitance can almost be eliminated. By properly adjusting the thicknesses and doping levels of the transport layer and the charge layer, the speed performance can be improved significantly because the drift time of electrons with saturation velocity is not a critical bandwidth-limiting factor compared with the multiplication time and response time of the velocity mismatch in long-absorption-length TWPDs [15]. As compared with the traditional APD, we can reduce its device capacitance by increasing the thickness of the photoabsorption layer; however, the tradeoff between the drift time of the photogenerated hole and the resistance-capacitance $(R C)$ bandwidth limitation will seriously limit its optimized speed performance [15], [32]. Our proposed APD structure also has the potential for improving the output-saturation-power-bandwidth-product performances [14] by increasing the device-absorption length [13], [15], [27] with less bandwidth degradation due to the reduction in device capacitance and microwave loss by incorporating the transport layer. Recently, by use of the diluted photoabsorption technique and evanescent edge-coupled structure, the high-power performance of SACM APD has been demonstrated in [28]. We employed the commercial computer-aided design (CAD) tool [38] to design and optimize the electrical-field distribution in the simulated structure. Fig. 2 shows the electric-field distribution for a well-designed APD with and without a charge layer. The thickness and doping density of each layer are shown in the inset table. By incorporating a charge layer, the operation voltage can be reduced significantly ( 25 versus $15 \mathrm{~V}$ ) under the same operation gain $\left(M_{e} \cong 10\right)$, and the magnitude of the electric field in the transport layer can be not only lower than that of the breakdown field $(\sim 300 \mathrm{kV} / \mathrm{cm})$, but also high enough $(>100 \mathrm{kV} / \mathrm{cm}$ ) to saturate the velocity of the electrons. The relationship between the gain $M_{e}$ and electric field will be discussed in details. The $\mathrm{p}^{+}$-graded doped photoabsorption $\mathrm{SL}$, which does not have an external applied electric field, has a built-in electric field to accelerate the diffusion process of photogenerated electrons and to avoid the breakdown problem.

\section{Bandwidth Simulation Model of the Device}

The propagation characteristics of photo-excited microwaves and optical waves in the traveling-wave structure are important with respect to our electrical bandwidth simulation. We modeled the electrical transmission line of this novel TWAPD using a hybrid-CPW-equivalent circuit model since its accuracy has been verified by the full-wave analysis technique [32]. The equivalent circuit model of the TWAPD structure is similar to the model of a traditional p-i-n TWPD, which is given in [32] and [39], except for the additional depletion capacitance and the serial impedance, which represent the multiplication layer and the charge layer, respectively. To simulate the optical waveguide in the TWAPD, we used the effective-index method to calculate the optical confinement factor and velocity. The optical refractive index of SiGe-Si SL $\left(n_{\mathrm{SLS}}\right)$ can be determined as follows [18]:

$$
n_{\mathrm{SLS}}^{2}=\left(\frac{d_{w}}{p}\right) n_{\mathrm{Si}}^{2}+\left(\frac{d_{b}}{p}\right) n_{\text {alloy }}^{2}
$$




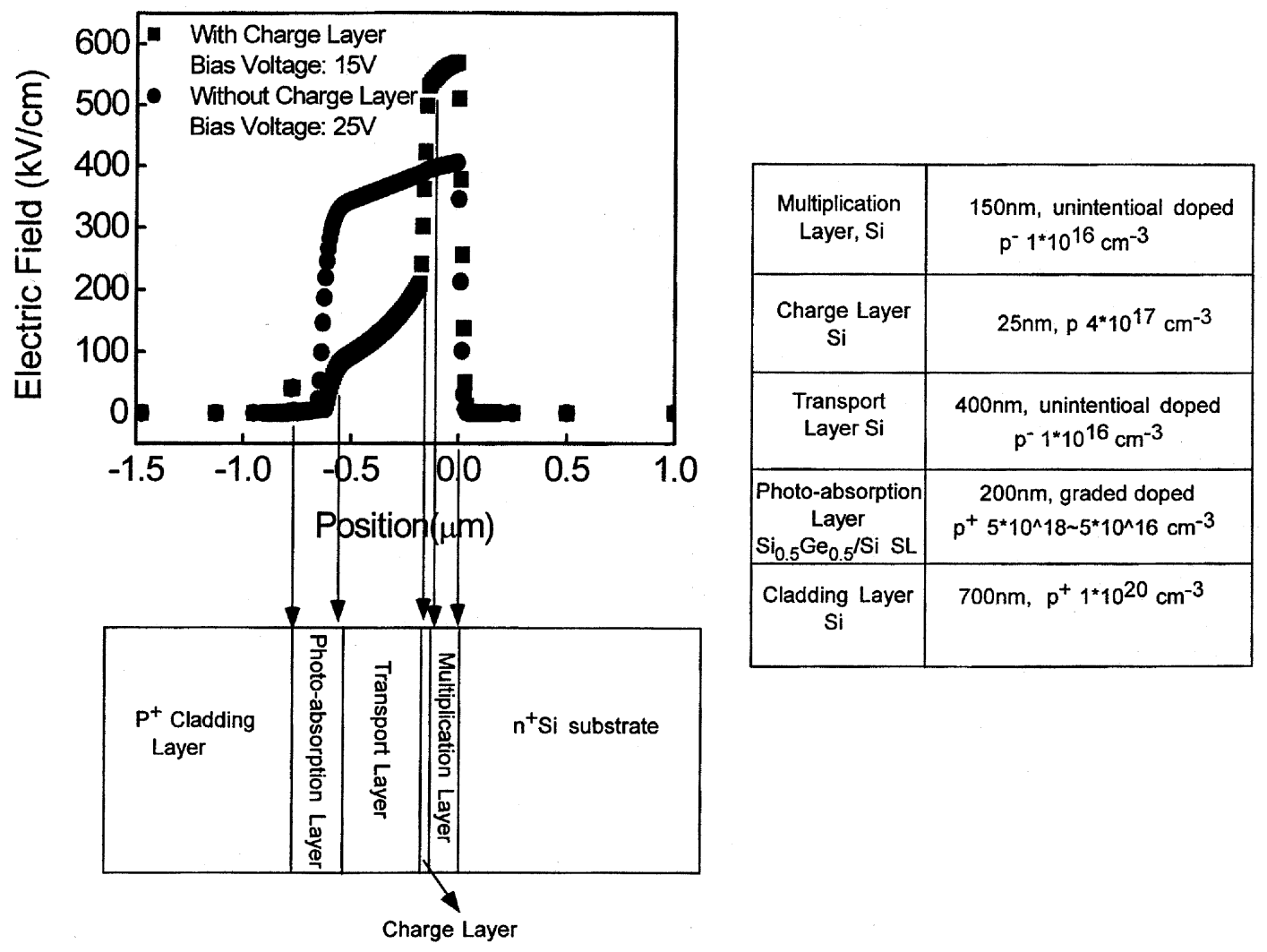

Fig. 2. Electric-field distributions versus positions in the structure of a SATCM APD with (squares) and without (circles) a charge layer.

where $d_{w}$ and $d_{b}$ are the thicknesses of the well and barrier, respectively, with a resulting SL period $p$ of $66 \AA$. The indexes of $\mathrm{Si}_{0.5} \mathrm{Ge}_{0.5}$ alloy $\left(n_{\text {alloy }}\right)$ and bulk $\mathrm{Si}\left(n_{\mathrm{Si}}\right)$ at a wavelength close to $1300 \mathrm{~nm}$ are 3.81 and 3.51, respectively [18], [40].

The velocity-mismatch bandwidth $B_{\mathrm{vm}}$ can be calculated using a photo-distributed current model with [15]

$$
\begin{aligned}
I_{f}(\omega)= & \exp \left(-\gamma_{m} l\right) \\
& \times\left(\frac{1-\exp \left(-\left(\gamma_{o}-\gamma_{m}\right) l\right)}{\gamma_{o}-\gamma_{m}}+\frac{1-\exp \left(-\left(\gamma_{o}+\gamma_{m}\right) l\right)}{\gamma_{o}+\gamma_{m}}\right)
\end{aligned}
$$

where $I_{f}(\omega)$ is the output photocurrent at the load end, which is a function of the microwave angular frequency $\omega . l$ is the device-absorption length. $\gamma_{m}$ is the microwave propagation constant, which can be obtained from the equivalent circuit model as discussed previously. $\gamma_{o}$ is the propagation constant of the photo-induced charge with

$$
\gamma_{o}=\Gamma \alpha_{o}+j \frac{\omega}{V_{o}}
$$

where $V_{o}, \alpha_{o}$, and $\Gamma$ are the optical group velocity, optical absorption constant of the photoabsorption layer, and optical confinement factor, respectively. The boundary reflection effect is a critical bandwidth-limiting factor, especially for the long-absorption-length TWPDs [15]. In order to model the bandwidth degradation caused by the load circuit mismatch effect, we can describe the total transmission efficiency using the formula [15]

$$
R_{b}(\omega)=\frac{T_{b}(\omega)}{1-\Gamma_{b}(\omega) \exp \left(-2 \gamma_{m} l\right)}
$$

where $\Gamma_{b}(\omega)=\left(Z_{l}-Z_{0}(\omega)\right) /\left(Z_{l}+Z_{0}(\omega)\right)$ is the reflection coefficient between the transmission line (with $Z_{0}(\omega)$ ) and load impedance (with $Z_{l}$ but without dispersion). $T_{b}(\omega)=2 Z_{l} /\left(Z_{l}+Z_{0}(\omega)\right)$ is the corresponding transmission coefficient. This equation is obtained by summing the endless boundary reflections between the open input end and the load end with maximum transmission normalized to 1 .

The multiplication time and transport time of photogenerated carriers also limit the speed performance of simulated TWAPDs. In our case, the transport time is dominated by the drift time of photogenerated electrons traveling through the $\mathrm{p}^{+}$-graded doped photoabsorption layer and the transport layer. The frequency response of the transport time [39] is calculated by

$$
I_{f}(\omega)=\left\{S a^{2}\left(\frac{t_{\mathrm{drift}}}{2}\right)+j \frac{2}{\omega t_{\mathrm{drift}}}\left[S a\left(\omega t_{\mathrm{drift}}\right)-1\right]\right\}
$$

where $S a\left(\omega t_{\text {drift }}\right) \equiv \sin \left(\omega t_{\text {drift }}\right) / \omega t_{\text {drift }} . t_{\text {drift }}$ is the total drift time of photogenerated electrons, which pass through the photoabsorption and transport layers. This expression can be derived by assuming triangular impulse photocurrent responses for electrons [39]. We assume that the applied electric field in the transport layer is high enough to saturate the velocity of photogenerated electrons, as shown in Fig. 2, and that the drift time $\left(t_{t}\right)$ in this layer can, thus, be obtained. The drift time $\left(t_{a}\right)$ of photogenerated electrons in a uniformly doped photoabsorption layer can be expressed as [41], [42]

$$
t_{a}=\frac{W_{a}^{2}}{2 D_{e}}+\frac{W_{a}}{v_{\mathrm{th}}}
$$


where $W_{a}$ is the thickness of the photoabsorption layer. $D_{e}$ and $v_{\text {th }}$ are the diffusion coefficient and thermal velocity of electrons in Si material, respectively. At room temperature, the value of $v_{\text {th }}$ is over $\sim 1 \times 10^{7} \mathrm{~cm} / \mathrm{s}$ [43], and the diffusion coefficient can be determined based on the mobility and Einstein relation. The mobility $\mu(x)$ can be obtained as follows [43]:

$$
\mu(x)=232+\frac{1180}{1+\left(\frac{N(x)}{8 \times 10^{16}}\right)^{0.9}}\left(\frac{\mathrm{cm}^{2}}{\mathrm{~V}-\mathrm{s}}\right) .
$$

$N(x)\left(\mathrm{cm}^{-3}\right)$ is the distribution of the dopant, which is a function of the depth " $x$ ". We assume a linear distribution of the acceptor-doping concentration, which varies from $10^{18}$ to $10^{16} \mathrm{~cm}^{-3}$.

By applying the graded doped technique to the photoabsorption layer, we can further reduce $t_{a}$ due to the built-in electric field [41]. This effect is considered in

$$
\frac{1}{t_{\text {anet }}}=\frac{1}{t_{a}}+\frac{1}{t_{\text {adrift }}}
$$

where $t_{\text {adrift }}$ and $t_{\text {anet }}$ represent, respectively, the drift time of photogenerated electrons under a built-in electric field and the net transport time, which includes the drift and diffusion processes in the graded doped photoabsorption layer. $t_{\text {adrift }}$ can be determined by [43]

$$
\begin{aligned}
E(x) & =\frac{k T}{q} \frac{1}{N(x)} \frac{d N(x)}{d x} \\
v(x) & =\mu(x) \times E(x) \\
t_{\text {adrift }} & =\int_{0}^{W_{a}} \frac{d x}{v(x)}
\end{aligned}
$$

where $v(x)$ is the velocity of electrons. We assume that the maximum velocity, calculated using (9), is the saturation velocity $\left(1 \times 10^{7} \mathrm{~cm} / \mathrm{s}\right)$ of electrons in Si. Because the mobility is a function of thickness as shown in (9), we have to modify the term $W_{a}^{2} / 2 D_{e}$ in (6) as follows [43]:

$$
t_{a}=\int_{0}^{W_{a}} \frac{\left(W_{a}-x\right) d x}{D_{e}(x)}+\frac{W_{a}}{v_{\mathrm{th}}} .
$$

In order to simulate the avalanche gain $\left(M_{e}\right)$ and multiplication time $\left(t_{m}\right)$, we have to determine the impact ionization coefficients of each electron $(\alpha)$ and hole $(\beta)$. The values for these coefficients can be obtained using [19]

$$
\begin{aligned}
& \alpha=1.04 \times 10^{6} \exp \left(\frac{-1.08 \times 10^{6}}{E}\right) \\
& \beta=0.45 \times 10^{6} \exp \left(\frac{-1.97 \times 10^{6}}{E}\right)
\end{aligned}
$$

where $E$ is the electric field in units of volts per centimeter. Equation (11) is obtained for silicon using a "reach-through" structure similar to a SAM APD [19]. $M_{e}$ can thus be obtained by [19]

$$
M_{e}=\frac{(\alpha-\beta) e^{d_{m}(\alpha-\beta)}}{\alpha-\beta e^{d_{m}(\alpha-\beta)}}
$$

where $d_{m}$ is the thickness of the multiplication layer. This gain equation is only valid for electrons injected into a multiplication

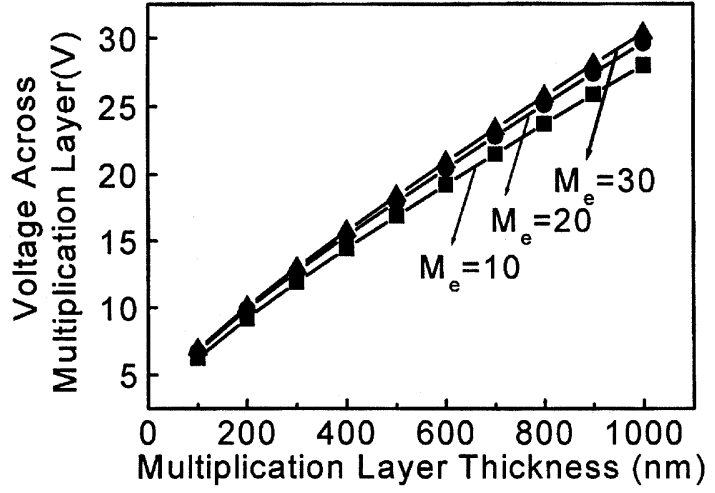

Fig. 3. Thickness of the multiplication layer versus the voltage across the multiplication layer for different desired operation gains $\left(M_{e}\right)$. Square: $M_{e}=$ 10. Circle: $M_{e}=20$. Triangle: $M_{e}=30$.

layer. The gain for hole-initiated avalanche breakdown can be obtained by simply exchanging $\alpha$ and $\beta$. Through the use of (11) and (12), the multiplication time can be obtained as follows [44]:

$$
\begin{aligned}
& t_{M}=N \times\left(\frac{\beta}{\alpha}\right) \times t_{m} \times M_{e}, \quad M_{e}>\frac{\alpha}{\beta} \\
& t_{M} \cong t_{m}, \quad M_{e} \leq \frac{\alpha}{\beta}
\end{aligned}
$$

where $N$ is a number varying slowly from $1 / 3$ to 2 as $\beta / \alpha$ varies from 1 to $10^{-3}$ [44]. $t_{m}$ is the multiplication-region transit time, which can be expressed as $d_{m} / v_{\mathrm{sat}}$. $v_{\mathrm{sat}}$ is the saturation velocity of electrons in $\mathrm{Si}$. The frequency response of the multiplication process $M(\omega)$ can be expressed as [45]

$$
M(\omega)=\frac{1}{\sqrt{1+\left(\omega t_{M}\right)^{2}}} .
$$

By multiplying (2), (4), (5), and (14), the net frequency response can be obtained [45].

\section{Design ConcePts AND BANDwidTh Simulation}

By using the bandwidth simulation model discussed previously, we can analyze and design the epilayer structure of this novel TWAPD. Fig. 3 shows the required bias voltage across the multiplication layer versus $d_{m}$ to obtain the desired $M_{e}$. Reducing the thickness of the multiplication layer can reduce the operating voltage of APDs, but a thin multiplication layer will usually result in a huge amount of dark current. The minimum thickness of the multiplication layer is $100 \mathrm{~nm}$ for high-speed operation with a reasonably low dark current [29].

The thickness of the photoabsorption layer determines the optical confinement factor $\Gamma$ and device-absorption length. In order to get a shorter device-absorption length and a higher velocity-mismatch bandwidth limit, a thick SiGe layer is required. Nevertheless, the critical thickness of the SiGe layer on the Si substrate limits the maximum thickness of the SiGe layer. On the other hand, the built-in electric field, which is caused by graded doping in this layer, will decrease when the thickness of the absorption layer increases. The tradeoff between the drift time in the photoabsorption layer and the device-absorption length must be optimized in the TWAPD design. Fig. 4 shows the net bandwidth versus the thickness of the photoabsorption layer under different total intrinsic layer (multiplication 


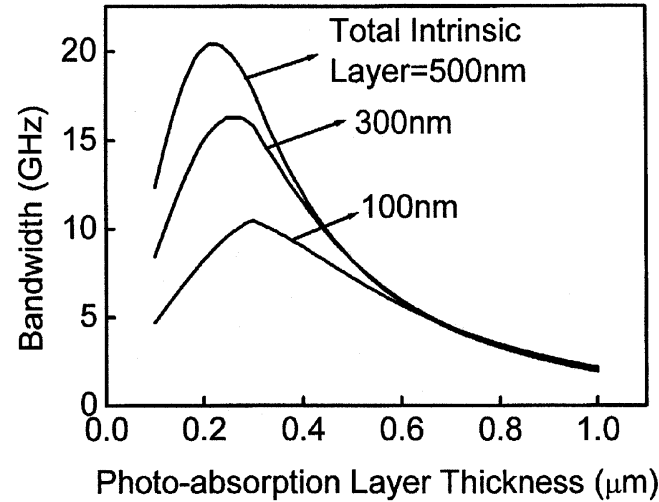

Fig. 4. Thickness of the photoabsorption layer versus the electrical bandwidth for different thicknesses of the intrinsic layer (multiplication layer with transport layer).

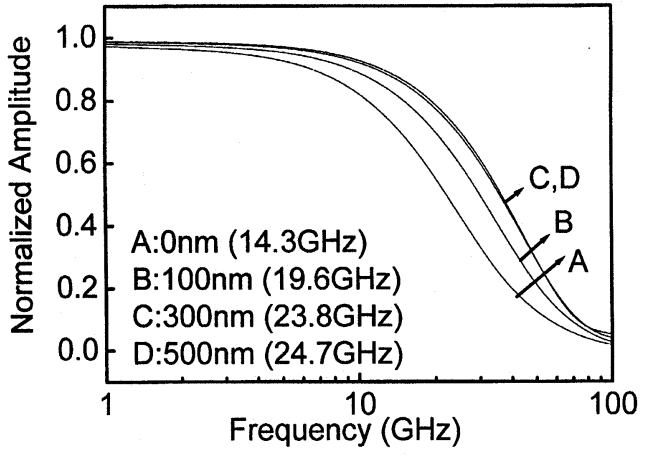

Fig. 5. Net frequency responses without consideration of the boundary reflection effect under different thicknesses of the transport layer. The thicknesses of traces A D are 0,100,300, and $500 \mathrm{~nm}$, respectively.

layer and transport layer) thicknesses. The device is simulated with $M_{e}=1$ (without gain), and its device-absorption length is assumed to be $1.5 / \Gamma \alpha_{o}$ [2]. We can clearly see that the optimum absorption-layer thickness slightly varies from $\sim 300$ to $\sim 200 \mathrm{~nm}$ when the thickness of the intrinsic layer increases from 100 to $500 \mathrm{~nm}$. A thick intrinsic layer implies a higher microwave velocity and higher velocity-mismatch bandwidth limit [15]. Thus, properly reducing the optical confinement factor, increasing the device-absorption length, and reducing the transport time of photogenerated electrons in the absorption layer can optimize the speed performance.

The thicknesses of the multiplication and transport layers seriously affect the electrical bandwidth and operation voltage of the SATCM TWAPD. Fig. 5 shows the simulated frequency response of a TWAPD with different thicknesses of the transport layer. The thickness of the multiplication layer is $100 \mathrm{~nm}$. The thickness of photoabsorption layer is $200 \mathrm{~nm}$ with a corresponding device-absorption length $\left(1.5 / \Gamma \alpha_{o}\right) 300 \mu \mathrm{m}$. The operation gain $M_{e}$ is fixed at 10 . We assume a perfect match load, which is integrated with the simulated TWAPD and that the boundary reflection effect can, thus, be neglected. The electrical bandwidth improves significantly $(\sim 14$ to $\sim 25 \mathrm{GHz})$ as the thickness of the transport layer increases. In practical applications, impedance mismatch between the external load and TWPD usually occurs due to size-difference-induced reflection [15] or the imaginary part of the characteristic impedance of a p-i-n-based TWPD. Serious bandwidth degradation due

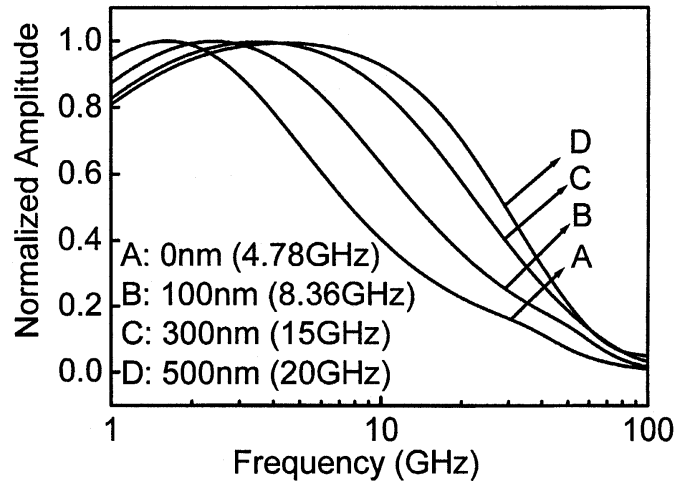

Fig. 6. Net frequency response with consideration of the boundary reflection effect for different thicknesses of the transport layer. The thicknesses of traces $\mathrm{A} \sim \mathrm{D}$ are $0,100,300$, and $500 \mathrm{~nm}$, respectively.

to the boundary reflection effect, especially for the long-absorption-length TWPDs, is thus unavoidable [15]. The characteristic impedance of the TWADP increases significantly due to the incorporation of a transport layer. Higher characteristic impedance can support better impedance matching between the external load and TWPD and results in less electrical bandwidth degradation [15]. Fig. 6 shows the frequency response of the TWAPD with different thicknesses of the transport layer and consideration of the boundary reflection effect. We assume a standard 50- $\Omega$ load at the output end of the TWAPD. Compared with Fig. 5, more significant bandwidth enhancement $(\sim 4 \times$ versus $\sim 1.7 \times$ ) can be observed.

The GBP is one of the most important figures of merit for APDs. Through the use of this parameter, we can further determine the optimum thicknesses of transport and multiplication layers. Fig. 7(a) shows the optimum thickness of the multiplication layer for maximum GBP value. The thickness of the photoabsorption layer is $200 \mathrm{~nm}$ with corresponding deviceabsorption length $\left(1.5 / \Gamma \alpha_{o}\right) 300 \mu \mathrm{m}$. The maximum voltage for each trace is selected such that the multiplication gain $M_{e}$ will be 60. Traces $\mathrm{A} \sim \mathrm{F}$ represent different thicknesses of the transport layer. When the thickness of the transport layer is less than $200 \mathrm{~nm}$, an optimum thickness of the multiplication layer for the maximum GBP value exists because, for a long-absorption-length TWAPD with an extremely thin total intrinsic layer (multiplication layer and transport layer), the dominant bandwidth-limiting factor is the boundary reflection or the velocity mismatch, not the multiplication time. Because of the increase of the multiplication layer thickness, the improvement of the characteristic impedance, microwave velocity, and GBP can thus be expected [15]. In Fig. 7(b), we plot the thickness of the transport layer versus GBP to determine the optimum thickness of the transport layer. Traces A $\sim$ E represents different thicknesses of the multiplication layer. With a thin multiplication layer $(100 \mathrm{~nm})$, as shown in trace A, GBP increases significantly with the thickness of the transport layer, and its optimum thickness is about $500 \mathrm{~nm}$. A thick transport layer can reduce the value of device capacitance, velocity mismatch, and the bandwidth limitation in boundary reflection effect significantly, especially in the case of TWAPD with a thin multiplication layer, which represents a large device capacitance.

Fig. 8 shows the frequency responses due to different bandwidth-limiting factors in a well-designed SATCM TWAPD. 

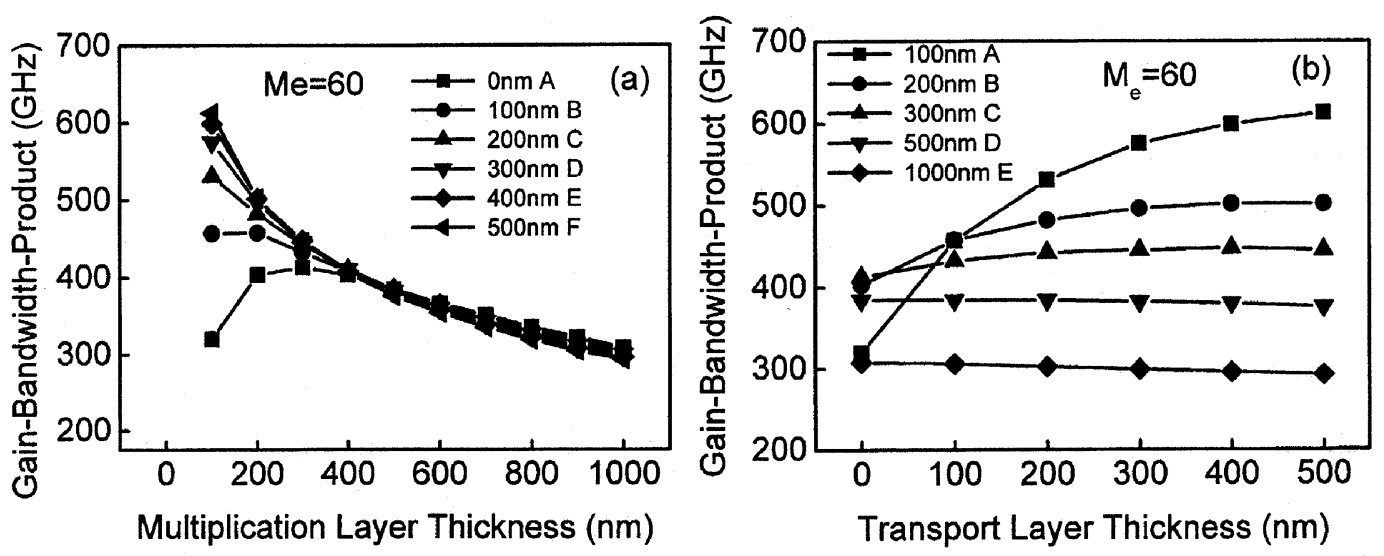

Fig. 7. GBP versus the (a) multiplication layer and (b) transport layer thickness. Each trace in (a) and (b) represents different transport layer and multiplication layer thicknesses, respectively. The operation gain $\left(M_{e}\right)$ of each simulated data point is fixed at 60 .

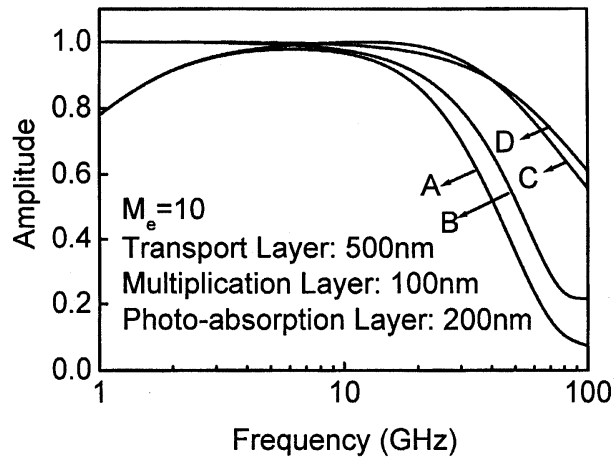

Fig. 8. Frequency responses due to different bandwidth-limiting factors for a well-designed SATCM TWAPD. Trace A: Net frequency response. Trace B: Carrier drift time. Trace C: Velocity mismatch with microwave loss and the boundary reflection effect. Trace D: Multiplication time.

The important parameters of this simulated device are labeled in this figure. We choose that the value of device-absorption length is equal to $150 \mu \mathrm{m}$ for the maximum bandwidth-responsivity product (quantum efficiency $\times$ bandwidth $\times$ gain). We can clearly see that the dominant bandwidth limitation, such as the velocity mismatch or microwave loss, in the traditional p-i-n-based TWPD with a long device-absorption length, is minimized in our structure. The speed performance of our simulated structure can thus be further improved by increasing the built-in electric field in the doped SiGe-Si SL photoabsorption layer, which can be achieved using a graded bandgap [43] or chirp SL technique [46].

\section{CONCLUSION}

This paper has proposed a novel type of avalanche photodetector: the SATCM TWAPD, which can improve the GBP and output-power-bandwidth product and reduce the operation voltage of traditional APDs. The analysis and bandwidth simulation presented in this paper have focused on a general example of an edge-coupled traveling-wave structure based on $\mathrm{Si}$ and $\mathrm{SiGe}$ materials due to its excellent breakdown property and high-speed/power applications in the communication wavelength. A photo-distributed current model, which includes the microwave propagation effects, transport time, and multiplication time of photogenerated carriers, has been used to conduct the simulation. According to our calculations, the electrical bandwidth and microwave propagation characteristics of the simulated TWAPD can be improved significantly almost without affecting the operation voltage by means of an additional transport layer. In addition, with a heavily doped photoabsorption layer and a large $\alpha / \beta$ ratio in the Si multiplication layer, we can almost eliminate the hole-transport and photoabsorption layer breakdown problems in the traditional APD structure and achieve a high-GBP performance. In a well-designed Si-SiGe-based SATCM TWAPD with a thin multiplication layer, relatively thick transport layer, and a long device-absorption length, the bandwidth-limiting factors, such as the velocity mismatch and the multiplication time, can be minimized, and the ultra-high-output-power-bandwidth-product performance can also be expected due to its small optical modal photoabsorption constant, the low density of photogenerated carriers, the large photoabsorption volume, and the superior microwave-guiding structure. The electrical bandwidth can be further increased by applying a bandgap engineering technique to the doped photoabsorption layer to shorten the drift time of photogenerated electrons.

\section{REFERENCES}

[1] A. Davidson and K. L. Dessau, "High-speed detectors: Faster measurements for today's laboratory," Photonics Spectra, pp. 110-114, Sept. 1998.

[2] K. Kato, "Ultrawide-band/high-frequency photodetectors," IEEE Trans. Microwave Theory Tech., vol. 47, pp. 1265-1281, July 1999.

[3] H. Nie, K. A. Anselm, C. Lenox, P. Yuan, C. Hu, G. Kinsey, B. G. Streetman, and J. C. Campbell, "Resonant-cavity separate absorption, charge and multiplication avalanche photodiodes with high-speed and high gain-bandwidth product," IEEE Photon. Technol. Lett., vol. 10, pp. 409-411, Mar. 1998.

[4] G. S. Kinsey, J. C. Campbell, and A. G. Dentai, "Waveguide avalanche photodiode operating at $1.55 \mu \mathrm{m}$ with a gain-bandwidth product of 320 GHz," IEEE Photon. Technol. Lett., vol. 13, pp. 842-844, Aug. 2001.

[5] J. Wei, J. C. Dries, H. Wang, M. L. Lange, G. H. Olsen, and S. R. Forrest, "Optimization of $10-\mathrm{Gb} / \mathrm{s}$ long-wavelength floating guard ring InGaAs-InP avalanche photodiodes," IEEE Photon. Technol. Lett., vol. 14, pp. 977-979, July 2002.

[6] J. Wei, F. Xia, and S. R. Forrest, "A high-responsivity high-bandwidth asymmetric twin-waveguide coupled InGaAs-InP-InAlAs avalanche photodiode," IEEE Photon. Technol. Lett., vol. 14, pp. 1590-1592, Nov. 2002.

[7] W. Wu, A. R. Hawkins, and J. E. Bowers, "Design of silicon hetero-interface photodetectors," J. Lightwave Technol., vol. 15, pp. 1608-1615, Aug. 1997. 
[8] Y. Kang, P. Mages, A. R. Clawson, P. K. L. Yu, M. Bitter, Z. Pan, A. Pauchard, S. Hummel, and Y. H. Lo, "Fused InGaAs-Si avalanche photodiodes with low-noise performances," IEEE Photon. Technol. Lett., vol. 14, pp. 1593-1595, Nov. 2002.

[9] S. Wang, R. Sidhu, X. G. Zheng, X. Li, X. Sun, A. L. Holmes Jr., and J. C. Campbell, "Low-noise avalanche photodiodes with graded impact-ionization-engineered multiplication region," IEEE Photon. Technol. Lett., vol. 13, pp. 1346-1348, Dec. 2001.

[10] S. Wang, F. Ma, X. Li, R. Sidhu, X. G. Zheng, X. Sun, A. L. Holmes Jr., and J. C. Campbell, "Ultra-low noise avalanche photodiodes with a 'centered-well' multiplication region," IEEE J. Quantum Electron., vol. 39, pp. 375-378, Feb. 2003.

[11] S. Jasmin, N. Vodjdani, J. Renaud, and A. Enard, "Diluted- and distributed-absorption microwave waveguide photodiodes for high efficiency and high power," IEEE Trans. Microwave Theory Tech., vol. 45, pp. 1337-1341, Aug. 1997.

[12] S. Murthy, T. Jung, M. C. Wu, Z. Wang, and W. Hsin, "Linearity improvement in photodetectors by using index-matching layer extensions," in Proc. IEEE Lasers \& Electro-Optics Soc. 2002 Annu. Meeting, Nov. 2002, pp. 424-425.

[13] V. M. Hietala, G. A. Vawter, T. M. Brenan, and B. E. Hammons, "Traveling-wave photodetectors for high-power, large bandwidth applications," IEEE Trans. Microwave Theory Tech., vol. 43, pp. 2291-2297, Sept. 1995

[14] J.-W. Shi, K. G. Gan, Y.-H. Chen, C.-K. Sun, Y. J. Chiu, and J. E. Bowers, "Ultra-high power-bandwidth product and nonlinear photo-conductance performances of low-temperature-grown GaAs based metal-semiconductor-metal traveling-wave photodetectors," IEEE Photon. Technol. Lett., vol. 14, pp. 1587-1589, Nov. 2002.

[15] J.-W. Shi and C.-K. Sun, "Design and analysis of long absorption length traveling wave photodetector," J. Lightwave Technol., vol. 18, pp. 2176-2187, Dec. 2000

[16] Y. J. Chiu, S. B. Fleischer, and J. E. Bowers, "High-speed low-temperature-grown GaAs p-i-n traveling-wave photodetector," IEEE Photon. Technol. Lett., vol. 10, pp. 1012-1014, July 1998.

[17] Z. Pei, L. S. Lai, H. P. Hwang, Y. T. Tseng, C. S. Liang, and M.-J. Tsai, " $\mathrm{Si}_{1-\mathrm{x}} \mathrm{Ge}_{\mathrm{x}}$ multi-quantum well phototransistor for near-infrared operation," Physica E: Low-Dimensional Systems Nanostructure, vol. 16, pp. 554-557, Mar. 2003.

[18] H. Temkin, A. Antreasyan, N. A. Olsson, T. P. Pearsall, and J. C. Bean, " $\mathrm{Ge}_{0.6} \mathrm{Si}_{0.4}$ rib waveguide avalanche photodetectors for $1.3 \mu \mathrm{m}$ operation," Appl. Phys. Lett., vol. 49, pp. 809-811, Sept. 1986.

[19] A. R. Hawkins, "Silicon-indium-gallium-arsenide avalanche photodetectors," Ph.D. dissertation, Dept. of Electrical and Computer Engineering, Univ. of California, Santa Barbara, CA, 1998.

[20] C. Li, Q. Yang, H. Wang, J. Yu, Q. Wang, Y. Li, J. Zhou, H. Huang, and X. Ren, "Back-incident SiGe-Si multiple quantum-well resonantcavity-enhanced photodetectors for 1.3- $\mu \mathrm{m}$ operation," IEEE Photon. Technol. Lett., vol. 12, pp. 1373-1375, Oct. 2000.

[21] H. Temkin, T. P. Pearsall, J. C. Bean, R. A. Logan, and S. L. Luryi, " $\mathrm{Ge}_{\mathrm{x}} \mathrm{Si}_{1-\mathrm{x}}$ strained-layer superlattice waveguide photodetectors operating near $1.3 \mu \mathrm{m}$," Appl. Phys. Lett., vol. 48, pp. 963-965, Apr. 1986.

[22] A. Splett, T. Zinke, K. Petermann, E. Kasper, H. Kibbel, H.-J. Herzog, and H. Presting, "Integration of waveguides and photodetectors in SiGe for $1.3 \mu \mathrm{m}$ operation," IEEE Photon. Technol. Lett., vol. 6, pp. 59-61, Jan. 1994.

[23] F. Y. Huang, K. Sakamoto, K. L. Wang, P. Trinh, and B. Jalali, "Epitaxial $\mathrm{SiGeC}$ waveguide photodetector on $\mathrm{Si}$ substrate with response in the 1.3-1.55 $\mu \mathrm{m}$," IEEE Photon. Technol. Lett., vol. 9, pp. 229-231, Feb. 1997.

[24] B. Li, G. Li, E. Liu, Z. Jiang, J. Qin, and X. Wang, "Monolithic integration of a $\mathrm{SiGe} / \mathrm{Si}$ modulator and multiple quantum well photodetector for $1.55 \mu \mathrm{m}$ operation," Appl. Phys. Lett., vol. 73, pp. 3504-3505, Dec. 1998.

[25] D. Buca, S. Winnerl, S. Lenk, C. Buchal, and D.-X. Xu, "Fast time response from $\mathrm{Si}-\mathrm{SiGe}$ undulating layer supperlatices," Appl. Phys. Lett., vol. 80, pp. 4172-4174, June 2002.

[26] H. Lafontaine, N. L. Rowell, S. Janz, and D.-X. Xu, "Growth of undulating $\mathrm{Si}_{0.5} \mathrm{Ge}_{0.5}$ layers for photodetectors at $\lambda=1.55 \mu \mathrm{m}$," J. Appl. Phys., vol. 86, pp. 1287-1291, Aug. 1999.

[27] J.-W. Shi, Y.-H. Chen, K.-G. G, Y.-J. Chiu, C.-K. Sun, and J. E. Bowers, "High speed and high power performances of LTG-GaAs based metalsemiconductor-metal traveling-wave-photodetectors in $1.3 \mu \mathrm{m}$ wavelength regime," IEEE Photon. Technol. Lett., vol. 14, pp. 363-365, Mar. 2002.

[28] S. Demiguel, X. Zheng, N. Li, X. Li, J. C. Campbell, J. Decobert, N. Tscherptner, and A. Anselm, "High-responsivity and high-speed evanescently coupled avalanche photodiodes," Electron. Lett., vol. 39, Dec. 2003.
[29] T. Nakata, T. Takeuchi, I. Watanabe, K. Makita, and T. Torikai, "10 Gbit/s high sensitivity, low-voltage-operation avalanche photodiodes with thin InAlAs multiplication layer and waveguide structure," Electron. Lett., vol. 36, pp. 2033-2034, Nov. 2000.

[30] S. Hanatani, M. Shishikura, S. Tanaka, H. Kitano, T. Miyazaki, and H. Nakamura, "A strained InAlAs/InGaAs superlattice avalanche photodiode for operation at an IC-power-supply voltage," in Proc. Int. Conf. Indium Phosphide Related Materials 1995, May 1995, pp. 369-372.

[31] T. Nakata, T. Takeuchi, K. Makita, and T. Torikai, "High-speed and high-sensitivity waveguide InAlAs avalanche photodiodes for 10-40 Gb/s receivers," in Proc. IEEE Lasers \& Electro-Optics Soc. 2001 Annu. Meeting, Nov. 2001, pp. 770-771.

[32] K. S. Giboney, M. J. W. Rodwell, and J. E. Bowers, "Traveling-wave photodetector theory," IEEE Trans. Microwave Theory Tech., vol. 45, pp. 1310-1319, Aug. 1997.

[33] Z. Pei, private communication.

[34] E. Quinones, S. K. Ray, K. C. Liu, and S. Banerjee, "Enhanced mobility PMOSFET's using tensile-strained $\mathrm{Si}_{1-\mathrm{y}} \mathrm{C}_{\mathrm{y}}$ layers," IEEE Electron Device Lett., vol. 20, pp. 338-340, July 1999.

[35] X. G. Zheng, J. S. Hsu, X. Sun, J. B. Hurst, X. Li, S. Wang, A. L. Holmes, J. C. Campbell, A. S. Huntington, and L. A. Coldren, "A $12 \times 12 \mathrm{In}_{0.53} \mathrm{Ga}_{0.47} \mathrm{As} \mathrm{In}_{0.52} \mathrm{Al}_{0.48}$ As avalanche photodiode array," IEEE J. Quantum Electron., vol. 38, pp. 1536-1540, Nov. 2002.

[36] C. Cohen-Jonathan, L. Giraudet, J. P. Praseuth, E. Legros, F. Heliot, and A. Bonzo, "Waveguide AlInAs/AlGaInAs avalanche photodiode for 20 Gbit/s photoreceivers," in Proc. Int. Conf. Indium Phosphide Related Materials 1997, May 1997, pp. 486-489.

[37] M. M. Hayat, O.-H. Kwon, Y. Pan, P. Sotirelis, J. C. Campbell, B. E. A. Saleh, and M. C. Teich, "Gain-bandwidth characteristics of thin avalanche photodiodes," IEEE Trans. Electron Devices, vol. 49, pp. 770-781, May 2002.

[38] DESSIS-ISE Release 7.5, Integrated Systems Engineering AG , Zurich, Switzerland, 2002.

[39] K. S. Giboney, "Traveling-wave photodetectors," Ph.D. dissertation, Dept. of Electrical and Computer Engineering, Univ. of California , Santa Barbara, CA, 1995.

[40] E. Kasper and K. Lyutovich, Properties of Silicon Germanium and SiGe:Carbon, INSPEC. London, U.K.: The Institution of Electrical Engineers Press, 2000.

[41] N. Shimizu, N. Watanabe, T. Furuta, and T. Ishibashi, "InP-InGaAs unitraveling-carrier photodiode with improved 3-dB bandwidth of over 150 GHz," IEEE Photon. Technol. Lett., vol. 10, pp. 412-414, Mar. 1998.

[42] — - "Bandwidth characteristics of InP/InGaAs uni-traveling-carrier photodiodes," in Tech. Dig. 1998 Int. Topical Meeting Microwave Photonic, Oct. 1998, pp. 193-194.

[43] Y. Taur and T. H. Ning, Fundamentals of Modern VLSI Devices. Cambridge, U.K.: Cambridge Univ. Press, 1998.

[44] R. B. Emmons, "Avalanche-photodiode frequency response," J. Appl. Phys., vol. 38, pp. 3705-3714, Aug. 1967.

[45] J. C. Campbell, W. S. Holden, G. J. Qua, and A. G. Dentai, "Frequency response of InP/InGaAsP/InGaAs avalanche photodiodes with separate absorption 'grading' and multiplication regions," IEEE J. Quantum Electron., vol. QE-21, pp. 1743-1746, Nov. 1985.

[46] C. Nguyen, T. Liu, M. Chen, H.-C. Sun, and D. Rensch, "AlInAs/GaInAs/InP double heterojunction bipolar transistor with a novel base-collector design for power application," IEEE Electron Device Lett., vol. 17, pp. 133-135, Mar. 1996.

Jin-Wei Shi, photograph and biography not available at the time of publication.

Yin-Hsin Liu, photograph and biography not available at the time of publication.

Chee-Wee Liu (M'99-SM'00), photograph and biography not available at the time of publication. 